\title{
Morphological and Compositional Analysis of Organic LEDs with Silicon QDs as Active Layer (SiLEDs)
}

\author{
Christian Kübel $^{1}$, Florian Maier-Flaig ${ }^{2}$, Julia Rinck ${ }^{3}$, Annie K. Powell ${ }^{3}$, Geoffrey A. Ozin ${ }^{4}$, U. Lemmer ${ }^{2}$ \\ 1. Institute of Nanotechnology (INT) and Karlsruhe NanoMicro Facility '(KNMF), Karlsruhe Insitute of \\ Technology (KIT), 76344 Eggenstein-Leopoldshafen, Germany \\ 2. Light Technology Institute (LTI) and DFG Center for Functional Nanostructures, Karlsruhe Institute \\ of Technology (KIT), Kaiserstraße. 12, 76131 Karlsruhe, Germany \\ 3. Institute of Inorganic Chemistry, Karlsruhe Institute of Technology, Engesserstraße 15, 76131 \\ Karlsruhe, Germany, Karlsruhe Institute of Technology, Kaiserstr. 12, 76131 Karlsruhe, Germany \\ ${ }^{4}$ Department of Chemistry, University of Toronto, 80 St. George Street, Toronto, ON, Canada M5S \\ 3H6 and DFG Center for Functional Nanostructures (CFN), Karlsruhe Institute of Technology, \\ Kaiserstr. 12, 76131 Karlsruhe, Germany.
}

Quantum dot light emitting diodes (QD-LEDs) feature great scientific and economic potential. Compared to their purely organic counterparts, the organic emissive layer of these devices is replaced by solution-processable functionalized quantum dots (QDs). QDs and QD-LEDs feature a wide spectral tunability and high color purity. Furthermore, as the efficiency of these devices is not intrinsically limited by spin statistics as in case of purely organic LEDs, they have the potential to reach much higher efficiencies. This, together with recently reported all-inorganic QD-LEDs without need for encapsulation, shows the great potential of this novel generation of LEDs in terms of efficiency and stability of the devices.

Even though a lot of work has been published on QD-LEDs, including the young field of silicon quantum dot based light emitting diodes (SiLEDs) [1], an in-depth study of the morphological properties of full devices before and after device operation is lacking in literature. Nevertheless, it is crucial to learn more about the degradation processes in order to further improve on the currently limited device life times. For purely organic LEDs, device failure is often related to migration or diffusion of elements forming non-conductive barriers, which lead to higher internal resistance and, as a consequence, destroy the device. However, no comparable data is available on the degradation properties related to morphological or compositional changes in QD-LEDs.

Here, we describe the results of a complete morphological and compositional study of SiLEDs using an image corrected FEI Titan 80-300 operated at 300kV. Initial BF-TEM and HAADF-STEM imaging was performed under strict low-dose conditions $\left(\right.$ dose $\left.<100 \mathrm{e} / \mathrm{nm}^{2}\right)$, but as the morphology of the organic multilayers is stable even at significantly higher doses, a more detailed compositional analysis by EFTEM and STEM-EDX mapping could be performed for the various 2.5-35 nm thick organic layers. The resulting elemental distribution for $\mathrm{C}, \mathrm{N}, \mathrm{O}, \mathrm{S}, \mathrm{Si}$ and $\mathrm{F}$ is in very good agreement with the nominal composition of the different layers (Fig. 1 and 2).

A comparison of as fabricated and electrically driven SiLEDs as well as SiLEDs prepared using monodisperse and polydisperse SiQDs [2] has been carried out to correlate morphological and compositional features with the degradation behavior and was combined with electroluminescence and photoluminescence life time studies. This analysis showed that the morphology and composition of the SiLED is very well preserved during normal operation of the devices even though the 
electroluminescence is reduced to $20 \%$ during this operation, which is attributed to atomic scale processes within the SiQDs themselves. In contrast, at high voltage/current, significant electromigration of SiQDs into the hole blocking layer TPBi is observed, whereas no change for the other organic layers is observed. For non-size separated SiQDs, device life times are significantly reduced compared to SiLEDs build from monodisperse SiQDs. This seems to be related to both percolating path of larger nanoparticles inside the SiQD layer as well as diffusion/electromigration of extremely small nanoparticles into the hole blocking layer. We expect that these results are not only valid for SiLEDs but also transferable to other QD-based LEDs.

\section{References:}

[1] F. Maier-Flaig, M. Stephan, T. Bocksrocker, M. Bruns, C. Kübel, J. Rinck, A.K. Powell, G.A. Ozin, U. Lemmer, Nano Lett., 13 (2013),475-480; DOI: 10.1021/n13038689.

[2] M. Mastronardi, F. Maier-Flaig, D. Faulkner, E. Henderson, C. Kübel, U. Lemmer, G.A. Ozin, Nano Lett., 12 (2012), 337-342; DOI: 10.1021/n12036194.
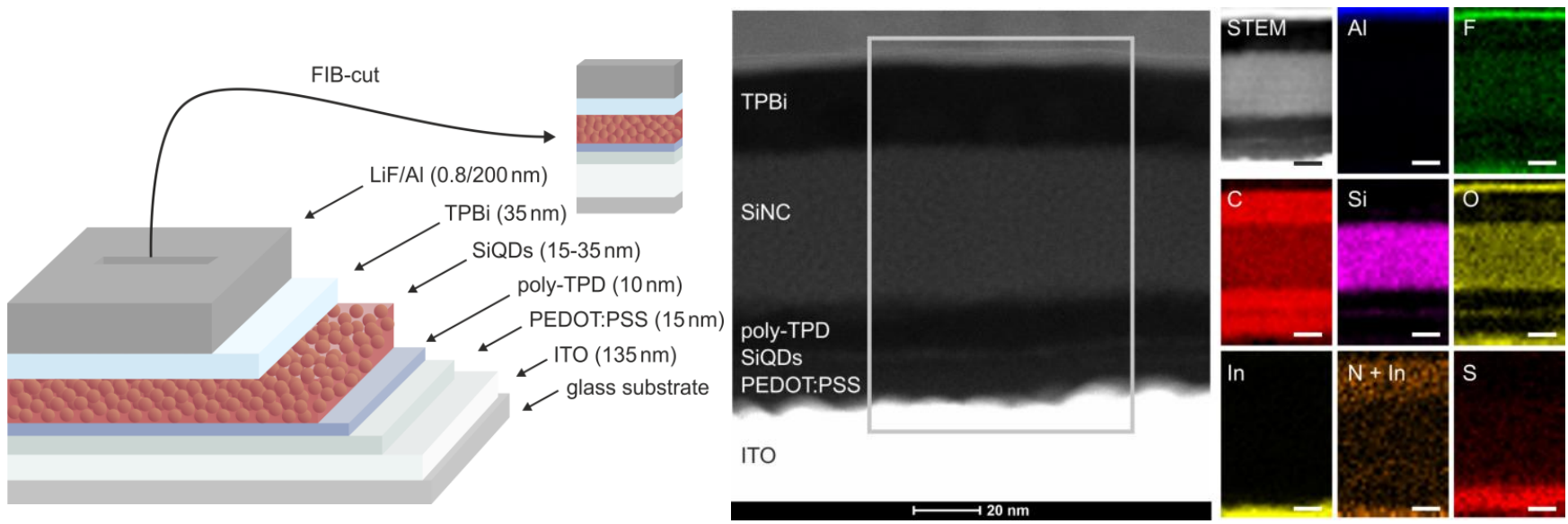

Figure 1. Schematic representation of the SiLED stack and HAADF-STEM cross-section image with corresponding EDX maps (scale bars $20 \mathrm{~nm}$ ).
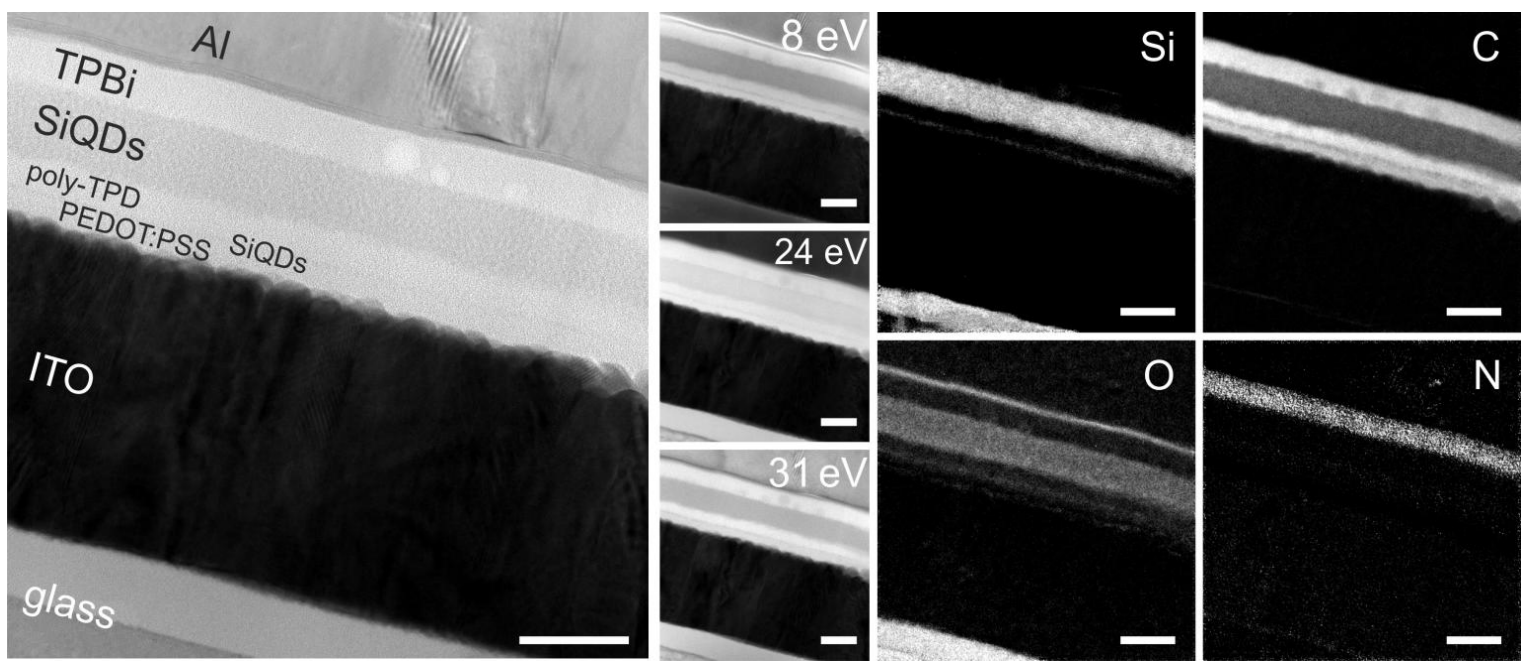

Figure 2. Zero-loss filtered BF-TEM image of a SiLED cross-section together with low-loss EFTEM images and elemental maps for silicon, carbon, oxygen and nitrogen (scale bars $50 \mathrm{~nm}$ ). 